\title{
Quasi phase reduction of all-to-all strongly coupled $\lambda-\omega$ oscillators near incoherent states
}

\author{
Iván León and Diego Pazó $\odot$ \\ Instituto de Física de Cantabria (IFCA), CSIC-Universidad de Cantabria, 39005 Santander, Spain
}

(Received 13 May 2020; accepted 10 September 2020; published 5 October 2020)

\begin{abstract}
The dynamics of an ensemble of $N$ weakly coupled limit-cycle oscillators can be captured by their $N$ phases using standard phase reduction techniques. However, it is a phenomenological fact that all-to-all strongly coupled limit-cycle oscillators may behave as "quasiphase oscillators," evidencing the need of novel reduction strategies. We introduce, here, quasi phase reduction (QPR), a scheme suited for identical oscillators with polar symmetry ( $\lambda-\omega$ systems). By applying QPR, we achieve a reduction to $N+2$ degrees of freedom: $N$ phase oscillators interacting through one independent complex variable. This "quasi phase model" is asymptotically valid in the neighborhood of incoherent states, irrespective of the coupling strength. The effectiveness of QPR is illustrated in a particular case, an ensemble of Stuart-Landau oscillators, obtaining exact stability boundaries of uniform and nonuniform incoherent states for a variety of couplings. An extension of QPR beyond the neighborhood of incoherence is also explored. Finally, a general QPR model with $N+2 M$ degrees of freedom is obtained for coupling through the first $M$ harmonics.
\end{abstract}

DOI: 10.1103/PhysRevE.102.042203

\section{INTRODUCTION}

Dynamical reduction is a concept of paramount importance in nonlinear dynamics [1], which may be used to reduce the number of degrees of freedom or to transform the evolution equations into a canonical form. Classical reduction techniques include adiabatic elimination [2], center-manifold reduction [3], and phase reduction [4-7]. The latter has been crucial to configure our comprehension of oscillatory media and coupled self-sustained oscillators.

Large ensembles of coupled self-sustained oscillators are found in a variety of domains ranging from biology and technology to the social sciences, see, e.g., Refs. [8-11] and references therein. It is well established that, if the coupling among $N$ limit-cycle oscillators is weak, then, phase reduction can be applied [4] and the dynamics becomes reliably described by $N$ phase oscillators. This approach yields a minimal description of emergent phenomena in all-to-all coupled oscillators as, for instance, collective synchronization [12-14], quasiperiodic partial synchronization (QPS) [15,16], or nonuniform incoherent states (NUISs) [17].

If the coupling is strong, however, phase reduction is not applicable as evidenced by several forms of collective chaos in globally coupled oscillators, which clearly elude phase reduction $[18,19]$. However, there are situations in which the oscillators, despite being strongly coupled, still resemble phase oscillators as their ordering on top of a closed curve is preserved in time. Straightforward examples are states in which the mean field vanishes such that each oscillator evolves as if it was uncoupled from the others. For identical oscillators, these states are called incoherent, or "phase-balanced configurations" if $N$ is finite [20]. The uniform incoherent state (UIS) - also called "splay state" for finite $N$-is the simplest form of incoherence. This was encountered a long time ago in arrays of Josephson junctions [21,22], populations of model neurons [23-25] (with the name of "asynchronous state"), and other systems. In contrast, other phase-balanced configurations, i.e., NUISs, have attracted much less attention. We are only aware that coexistence of different NUISs is nowadays being investigated in the context of some engineering applications [26,27]. Apart from incoherent states, there are more complex phenomena, such as QPS, modulated QPS, or pure collective chaos in which identical oscillators behave as "quasiphase oscillators" on top of an unsteady closed curve $[19,28,29]$. Recent advances extending standard phase reduction beyond the first order do not appear to be practical enough even to cover the moderate coupling regime $[17,30]$. Alternative methods based on phase-amplitude reduction or isostables fall short in the dimensionality reduction actually achieved [6,31-33].

In this paper, we present quasi phase reduction (QPR), a dynamical reduction method to capture the dynamics of allto-all coupled identical limit-cycle oscillators near incoherent states. For standard phase reduction, the zeroth order corresponds to tuning the coupling to zero. In our new approach, the incoherent states play the role of zeroth-order solutions, and the mean field will be the "small quantity" of our theory. Moreover, the number of oscillators is irrelevant, it may be either finite or infinite. The QPR method only covers identical $\lambda-\omega$ oscillators (two-dimensional systems with polar symmetry), but still, it is conceptually appealing since it yields a significant dimensionality reduction from $2 N$ to $N+2$ degrees of freedom. The reduced system consists of $N$ phase oscillators and one complex-valued variable. Thereupon we can calculate analytically the stability boundary of incoherent states. Moreover, we explore an extension of QPR, keeping the $N+2$ degrees of freedom, which correctly pinpoints a saddle QPS at moderate coupling in a specific model. Finally, 
general QPR with $N+2 M$ degrees of freedom is derived for coupling through the $M$ th harmonic. Throughout this paper, the correctness of our approach is confirmed by numerical simulations with a popular $\lambda-\omega$ system called the StuartLandau oscillator.

The paper is organized as follows. In Sec. II, we introduce the $\lambda-\omega$ oscillator and the isochrons. Incoherent states in a particular system of globally coupled $\lambda-\omega$ oscillators are reviewed in Sec. III for illustrative purposes. Section IV presents QPR for a family of coupling functions. The results in Sec. IV are applied to Stuart-Landau oscillators in Sec. V. Sections VI and VII extend the results in Sec. IV beyond the lowest order, and to other coupling functions, respectively. The conclusions are summarized in Sec. VIII.

\section{II. $\lambda-\omega$ OSCILLATOR}

In this paper, we restrict ourselves to oscillators of the $\lambda-\omega$ type $[9,34]$. These are two-dimensional systems with rotational symmetry, which admit the following representation of the evolution equations in polar coordinates:

$$
\begin{aligned}
& \dot{r}=\lambda(r) r, \\
& \dot{\phi}=\omega(r) .
\end{aligned}
$$

The overdot denotes the time derivative as usual. Without lack of generality, we assume the existence of a stable limit cycle at $r=1$, i.e., $\lambda(1)=0$. Moreover, the natural frequency of the oscillator is $\Omega=\omega(1)$. The attraction rate to the limit cycle is given by the second Floquet exponent $\Lambda=\left.\frac{d \lambda}{d r}\right|_{r=1}<$ 0 . Alternative to Eq. (1), we can work with the complex variable $A=r e^{i \phi}$ such that the $\lambda-\omega$ oscillator obeys

$$
\dot{A}=f(A) \text {, }
$$

where function $f$ satisfies $f\left(A e^{i \alpha}\right)=e^{i \alpha} f(A)$. For simplicity, it is convenient to assume that $f$ can be expressed as a series of the form

$$
f(A)=\sum_{n=-\infty}^{\infty} f_{n}|A|^{n} A,
$$

where $f_{n}$ are complex coefficients. The existence of an attractive limit cycle of frequency $\Omega$ implies $\sum_{n} n \operatorname{Re}\left(f_{n}\right)=\Lambda$ and $\sum_{n} f_{n}=i \Omega$. Common instances of $\lambda-\omega$ systems contain a small number of nonzero coefficients $f_{n}$ in Eq. (3). If only $f_{0}$ and $f_{2}$ are nonzero [with $\operatorname{Re}\left(f_{0}\right)=-\operatorname{Re}\left(f_{2}\right)>0$ ] we have the well-known Stuart-Landau oscillator [4], the normal form of a supercritical Hopf bifurcation. Adding other nonzero terms we get, for instance, the normal form of the generalized (Bautin) Hopf bifurcation if $f_{4} \neq 0$ [3], or the slow-amplitude dynamics of a parametric feedback oscillator as used in microand nanoelectromechanics if $f_{-1} \neq 0$ [35].

\section{A. Isochrons}

To account for the effect of perturbations, phase reduction approaches require extending the definition of the phase away from the limit cycle $[4,5,7,9]$. To do so, we seek a phase variable $\theta$ such that $\dot{\theta}=\Omega$ holds in the whole basin of attraction not only on the limit cycle. The isochron is defined as the set of points that converge to the same "asymptotic phase" on the limit cycle. For $\lambda-\omega$ systems, polar symmetry yields a relation between the phase $\theta$ and the polar coordinates of the form [9]

$$
\theta(r, \phi)=\phi-\chi(r),
$$

with $\chi(1)=0$. The phase dynamics satisfies $\dot{\theta}=\dot{\phi}-\frac{d \chi}{d r} \dot{r}$, and imposing $\dot{\theta}=\Omega$, we solve the equation for $\chi(r)$,

$$
\chi(r)=\int_{1}^{r} \frac{\omega(\hat{r})-\Omega}{\lambda(\hat{r}) \hat{r}} d \hat{r}=\int_{1}^{r} \frac{\sum_{n} \operatorname{Im}\left(f_{n}\right) \hat{r}^{n}-\Omega}{\sum_{n} \operatorname{Re}\left(f_{n}\right) \hat{r}^{n+1}} d \hat{r} .
$$

Depending on the specific oscillator type considered, a closed analytical solution of $\chi(r)$ may or may not exist. However, if deviations from the limit cycle are small, it is enough to know the first coefficient of the Taylor expansion of $\chi$ around $r=1$,

$$
\chi(1+\delta r)=\chi_{0} \delta r+O\left(\delta r^{2}\right)
$$

where $\chi_{0}=\left.\frac{d \chi}{d r}\right|_{r=1}$. Differentiating Eq. (5) and evaluating the limit $r \rightarrow 1$ by L'Hôpital's rule, we get

$$
\chi_{0}=\frac{\sum_{n} n \operatorname{Im}\left(f_{n}\right)}{\sum_{n}(n+1) \operatorname{Re}\left(f_{n}\right)}=\frac{\sum_{n} n \operatorname{Im}\left(f_{n}\right)}{\Lambda} .
$$

This expression together with $\sum_{n} n \operatorname{Re}\left(f_{n}\right)=\Lambda$, obtained before, can be cast in a compact form

$$
\sum_{n=-\infty}^{\infty} n f_{n}=\Lambda\left(1+i \chi_{0}\right)
$$

\section{B. Stuart-Landau oscillators}

In this paper, we asses the validity of our theoretical findings with the Stuart-Landau oscillator, which is a universal representation (via center-manifold reduction) of systems in the neighborhood of a Hopf bifurcation. It reads

$$
\dot{A}=A-\left(1+i c_{2}\right)|A|^{2} A \text {. }
$$

The limit cycle at $|A|=1$ has a second Floquet exponent $\Lambda=$ -2 . Moreover, the isochrons are logarithmic spirals $\theta=\phi-$ $c_{2} \ln r$, where $c_{2}$ in Eq. (7) is the so-called nonisochronicity parameter. Therefore, $\chi_{0}=c_{2}$ for this system.

\section{AN EXAMPLE: THE MEAN-FIELD COMPLEX GINZBURG-LANDAU EQUATION}

Before presenting the QPR method, it is instructive to recall a well-studied model of globally coupled $\lambda-\omega$ oscillators in which incoherent states are observed: The mean-field complex Ginzburg-Landau equation (MF-CGLE). It consists of $N$ diffusively coupled Stuart-Landau oscillators [18,19],

$$
\dot{A_{j}}=A_{j}-\left(1+i c_{2}\right)\left|A_{j}\right|^{2} A_{j}+\epsilon\left(1+i c_{1}\right)\left(\bar{A}-A_{j}\right),
$$

here, constants $\epsilon$ and $c_{1}$ determine the strength and the reactivity of the coupling, respectively; and $\bar{A}=\frac{1}{N} \sum_{k=1}^{N} A_{k}$. The MF-CGLE is a discretization of the complex GinzburgLandau equation on a fully connected lattice. The last term of Eq. (8) is a discrete version of the Laplacian on such a lattice. 

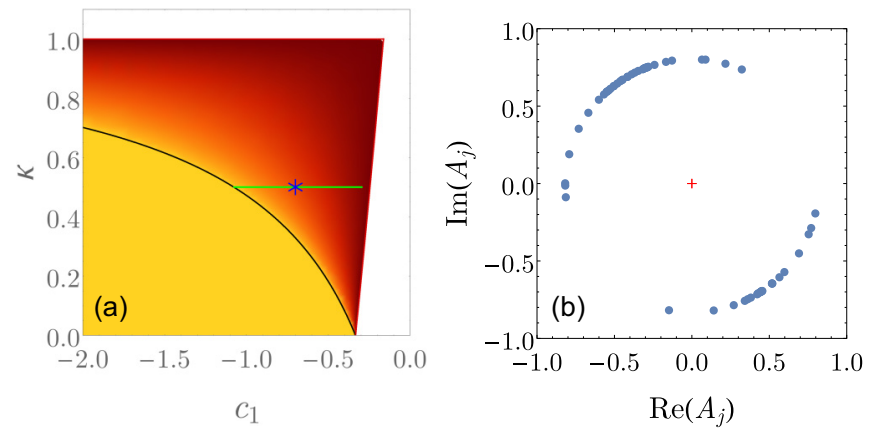

FIG. 1. (a) Partial phase diagram of the MF-CGLE (9) for $c_{2}=$ 3 , showing the domain of the UIS and NUISs in the presence of an infinitesimal noise. In the yellow region, the UIS is stable, whereas different NUISs are observed inside the other shaded region. The color shading codes the unevenness of the oscillator density through the value of $\left|Z_{2}\right| \equiv Q$. In the white region, all incoherent states are unstable. The asterisk and the green line indicate the parameter values used in panel (b) and in Fig. 3, respectively. (b) Snapshot of a random sample of 60 oscillators out of $N=300$, after a numerical simulation of $2 \times 10^{6}$ time units where an independent white Gaussian noise of intensity $D=10^{-6}$ along the real and imaginary parts of the $A_{j}$ 's has been added to remove the degeneracy among infinitely many neutrally stable NUISs.

The MF-CGLE is a prototype of system with many degrees of freedom and a rich repertoire of collective behaviors. In addition to full synchrony, UIS, NUISs, QPS, and clustering, the system displays several forms of chaos and has attracted considerable attention over the years [18,19,28,29,36-39].

For better comparison with the QPR theory, it is convenient to absorb the local term $\epsilon\left(1+i c_{1}\right) A_{j}$. Specifically, setting

$$
\kappa=\frac{\epsilon}{1-\epsilon}
$$

rescaling time $\left(t \rightarrow \frac{t}{1-\epsilon}\right)$, and going to a rotating frame with rescaled amplitude

$$
\begin{aligned}
& \left(A_{j} \rightarrow \frac{A_{j}}{\sqrt{1-\epsilon}} e^{-i\left(\epsilon c_{1}+c_{2}\right) t}\right), \text { we get } \\
& \quad \dot{A_{j}}=\left(1+i c_{2}\right)\left(1-\left|A_{j}\right|^{2}\right) A_{j}+\kappa\left(1+i c_{1}\right) \bar{A} .
\end{aligned}
$$

At variance with the Stuart-Landau Eq. (7), the linear coefficient has a nonzero imaginary part as we have adopted a rotating frame such that $\Omega=0$.

In a broad region of parameter space, the system (9) settles into an incoherent state, i.e., with zero mean field $\bar{A}=0$. This does not specify the state of the system as it holds for a continuum of oscillator arrangements for $N>3$. The most prominent incoherent state is the UIS, corresponding to oscillators located over a circle with uniformly distributed phases. For the remaining incoherent states, there is a lack of uniformity in the oscillator distribution, and we use the acronym NUIS for them. Figure 1(a) shows a partial phase diagram for Eq. (9) for a specific value of $c_{2}=3$. The UIS is observed in the light (yellow) shaded region at the left of the black solid line $[18,19]$. In the other shaded region, a NUIS settles spontaneously (the UIS is unstable). The asterisk in the phase diagram indicates the parameter values for the snapshot of the NUIS in Fig. 1(b). In this figure, it is apparent that the oscillators are not evenly distributed, whereas $\bar{A}$, represented by a red cross, settles at the origin.

\section{IV. $(N+2)$-QUASI PHASE REDUCTION}

In this section, we present our QPR method from $2 N$ to $N+2$ degrees of freedom for $N$ coupled $\lambda-\omega$ oscillators. The reduced system consists of $N$ phases plus two global degrees of freedom, hence, the name of QPR. The validity of the method requires a weak perturbation in the oscillators' motion, what holds in the neighborhood of the incoherent states, irrespective of the coupling strength.

\section{A. Coupling}

We will consider globally coupled identical oscillators,

$$
\dot{A_{j}}=f\left(A_{j}\right)+\kappa g(\mathcal{A})
$$

where $\kappa$ is a positive coupling constant, and $\mathcal{A}$ denotes one or more mean fields of the set $\mathcal{C}_{A}, \mathcal{A} \subseteq \mathcal{C}_{A}$. The set $\mathcal{C}_{A}$ of mean fields is as follows:

$$
\mathcal{C}_{A}=\left\{\overline{|A|^{n} A}\right\}_{n \in \mathbb{Z}} \cup\left\{\overline{|A|^{n} A^{*}}\right\}_{n \in \mathbb{Z}} .
$$

Here, $\overline{|A|^{n} A}=\frac{1}{N} \sum_{j=1}^{N}\left|A_{j}\right|^{n} A_{j}$ and * stands for complex conjugation. Note that only the first harmonic in $\phi$ enters in the interaction. (The case with higher harmonics in the coupling is discussed in Sec. VII). Moreover, we demand the interaction function $g$ in Eq. (10) to vanish when the mean fields in the argument vanish, i.e., $g(\mathcal{A}=0)=0$. Among the possible couplings the most preeminent one is diffusion, $g(\mathcal{A}) \propto \bar{A}$, as in the MF-CGLE (9) introduced above $[18,19]$. Other examples with nonlinear coupling as $g(\mathcal{A}) \propto \bar{A}+b|\bar{A}|^{2} \bar{A}$ and $g(\mathcal{A}) \propto \bar{A}+b \mid \overline{\left.A\right|^{2} A}$ have been considered in Refs. [40,41], respectively. It is also important to note that we do not exclude symmetry breaking terms in the coupling, such as $\operatorname{Re}(\bar{A})$, similar to Ref. [42].

\section{B. Preliminaries}

The first step of the analysis is to obtain the evolution equation for the dynamics of the phases. Equation (10) in polar coordinates becomes

$$
\begin{aligned}
\dot{r}_{j} & =\lambda\left(r_{j}\right) r_{j}+\kappa \operatorname{Re}\left[g(\mathcal{A}) e^{-i \phi_{j}}\right], \\
\dot{\phi}_{j} & =\omega\left(r_{j}\right)+\frac{\kappa}{r_{j}} \operatorname{Im}\left[g(\mathcal{A}) e^{-i \phi_{j}}\right] .
\end{aligned}
$$

The phase dynamics is obtained through the change of variables in (4),

$$
\dot{\theta}_{j}=\Omega+\frac{\kappa}{r_{j}} \operatorname{Im}\left\{\left[1-i r_{j} \chi^{\prime}\left(r_{j}\right)\right] g(\mathcal{A}) e^{-i\left[\theta_{j}+\chi\left(r_{j}\right)\right]}\right\},
$$

here $\chi^{\prime}\left(r_{j}\right)$ denotes the derivative of $\chi$ with respect to $r$ evaluated at $r_{j}$. In order to reduce the dimensionality of the system, we seek to remove the dependence on the radii $r_{j}$. Using Eq. (12a), we write the evolution equation for an infinitesimal perturbation $\delta r_{j}$ off the limit-cycle $\left(r_{j}=1+\delta r_{j}\right)$,

$$
\delta \dot{r}_{j}=\Lambda \delta r_{j}+\kappa \operatorname{Re}\left[g(\mathcal{A}) e^{-i \theta_{j}}\left(1-i \chi_{0} \delta r_{j}\right)\right]+O\left(\delta r_{j}^{2}\right)
$$

It is obvious that the oscillators will be in the proximity of the limit cycle whenever $\kappa|g(\mathcal{A})| \ll-\Lambda$. If this condition holds, 
we can set $r_{j}=1$ in (13), obtaining thereby the lowest-order approximation,

$$
\dot{\theta}_{j}=\Omega+\kappa \operatorname{Im}\left[\left(1-i \chi_{0}\right) g(\mathcal{A}) e^{-i \theta_{j}}\right] .
$$

This equation is not closed, as there are still dependences on the mean field(s) through $g(\mathcal{A})$.

\section{Small $\kappa:$ Standard first-order phase reduction}

To put our paper in context, and for later comparison, we note that traditional first-order phase reduction assumes $\kappa \ll$ $-\Lambda$, which automatically implies $\delta r_{j} \simeq 0$, as noted above. Therefore, the mean fields in $g(\mathcal{A})$ can be approximated as

$$
\overline{|A|^{n} A}=\overline{r^{n+1} e^{i[\theta+\chi(r)]}} \simeq Z,
$$

where $Z \equiv \overline{e^{i \theta}}$ is the Kuramoto order parameter. Thus, at the lowest order, the coupling term will only depend on $Z$. In this case, we can make the replacement $g(\mathcal{A}) \simeq \hat{\Gamma}(Z)$ in Eq. (15) obtaining

$$
\dot{\theta}_{j}=\Omega+\kappa \operatorname{Im}\left[\left(1-i \chi_{0}\right) \hat{\Gamma}(Z) e^{-i \theta_{j}}\right] .
$$

This system of $N$ phase oscillators is the first-order phase reduction of (10). This reduction works poorly if the coupling is not small; and, even for asymptotically small coupling, there are states of (10) not reproducible by Eq. (17), such as the NUIS [shown in Fig. 1(b)] or QPS. Higher-order terms proportional to $\kappa^{2}, \kappa^{3}$, etc., can be incorporated into (17) removing degeneracies and extending the validity of the phase model with $N$ degrees of freedom [17]. However, if the coupling is strong this procedure is either impractical (as the convergence rate of the series in powers of $\kappa$ is not fast enough [17]) or plain wrong (if the expansion in $\kappa$ is divergent).

\section{Small $|g(\mathcal{A})|$ : Quasi phase reduction of Eq. (10)}

Incoherent states, the starting point of our analysis, are configurations of the oscillators compatible with $\mathcal{A}=0$. Accordingly, in an incoherent state, each oscillator evolves as if it experienced no input from the rest of the population. An ensemble of identical oscillators may spontaneously settle into the UIS or NUISs in wide regions of parameter space, see, e.g., Fig. 1. Moreover, it is phenomenologically observed that there are also nonincoherent states in which strongly coupled oscillators behave as quasiphase oscillators [29], preserving their ordering on top of a closed curve that evolves in time. This occurs, in particular, in globally coupled Stuart-Landau oscillators when the UIS loses its stability giving rise to a state called the QPS which, after secondary instabilities, yields pure collective chaos [19,29].

With the aim at describing the previous phenomena in a minimal way, we resort to Eq. (15) since it already suggests that some kind of perturbative approach in small $g(\mathcal{A})$ is feasible in analogy to the small $\kappa$ approximation in standard phase reduction. As Eq. (15) is not closed due to $g(\mathcal{A})$, we are tempted to consider $g(\mathcal{A})$ as a new variable. This is not the best choice as the evolution equation cannot be generally closed in terms of $g(\mathcal{A})$. Instead, the complex variable $B=\bar{A}$ is the right choice since, as shown below, any mean field $\overline{|A|^{n} A}$ can be approximately expressed in terms of $B$ and $Z$. Assuming the proximity of the oscillators to their fiducial limit cycles, $r=1+\delta r$, we expand $\phi=\theta+\chi_{0} \delta r+O\left(\delta r^{2}\right)$. In this way, the mean field $B$ is

$$
B=\bar{A}=\overline{r e^{i \phi}} \simeq \overline{(1+\delta r) e^{i\left(\theta+i \chi_{0} \delta r\right)}} \simeq Z+\left(1+i \chi_{0}\right) \overline{\delta r e^{i \theta}} .
$$

Therefore, we can express the average $\overline{\delta r e^{i \theta}}$ in terms of $B$ and $Z$,

$$
\overline{\delta r e^{i \theta}} \simeq \frac{B-Z}{\left(1+i \chi_{0}\right)},
$$

and apply this identity to all the other mean fields, obtaining a linear dependence of $\overline{|A|^{n} A}$ on $B$ and $Z$,

$$
\begin{aligned}
\overline{|A|^{n} A} & =\overline{r^{n+1} e^{i \phi}} \\
& \simeq Z+\left(n+1+i \chi_{0}\right) \overline{\delta r e^{i \theta}} \\
& \simeq B+\frac{n}{1+i \chi_{0}}(B-Z) .
\end{aligned}
$$

With the previous equation, any $g(\mathcal{A})$ can be approximated by a function of $Z$ and $B$,

$$
g(\mathcal{A}) \simeq \Gamma(Z, B) .
$$

Now, the evolution of $B$ is obtained averaging (10) over the whole population, Namely,

$$
\dot{B}=\frac{1}{N} \sum_{k=1}^{N} \dot{A_{k}}=\overline{f(A)}+\kappa g(\mathcal{A}) .
$$

The term $\overline{f(A)}$ is calculated using Eqs. (3), (6), and (20),

$$
\overline{f(A)} \simeq i \Omega B+\Lambda(B-Z) .
$$

Finally, replacing Eqs. (21) and (23) into Eqs. (15) and (22) we obtain the $(N+2)$-QPR of the globally coupled oscillator system defined by Eq. (10),

$$
\begin{aligned}
\dot{\theta}_{j} & =\Omega+\kappa \operatorname{Im}\left[\left(1-i \chi_{0}\right) \Gamma(Z, B) e^{-i \theta_{j}}\right] \\
\dot{B} & =i \Omega B+\Lambda(B-Z)+\kappa \Gamma(Z, B) .
\end{aligned}
$$

These equations are the main result of this paper. Some important remarks follow.

\section{Remarks on the $(N+2)-Q P R, E q$. (24)}

The QPR that transforms (10) into (24) entails a drastic decrease in the number of degrees of freedom from $2 N$ to $N+2$ : one complex variable $B$ plus $N$ phases. In contrast to standard phase reduction, there is an extra complex variable $B$. This is the key ingredient to make the strong coupling amenable to analysis, whereas preserving the population of phase oscillators. The theory is consistent since QPR (24) boils down to the standard phase reduction (17) in the $\kappa \rightarrow 0$ limit. To see this, set $\Omega=0$ in (24) by going to a rotating frame $\left(\theta_{j}^{\prime}, B^{\prime}\right)=\left(\theta_{j}-\Omega t, B e^{-i \Omega t}\right)$ if necessary, and note that $B(t) \rightarrow Z(t)$ as $\kappa \rightarrow 0$ in Eq. (24b). In this way, Eq. (24a) reduces to $(17)$ since $\Gamma(Z, Z)=\hat{\Gamma}(Z)$, cf. Eqs. (16) and (20).

Equation (24) can be regarded as a population of phase oscillators coupled through a sort of external medium $B$. Indeed, a similar model is obtained applying ordinary phase reduction (assuming weak coupling) to a model of "dynamical quorum sensing" in which oscillators are coupled through a medium 
with intrinsic dynamics [43]. Here, in sharp contrast, there is no medium in the original system (10), instead, QPR endows the mean field with a virtual dynamical equation.

An important feature of Eq. (24) [as a consequence of the approximations (18) and (19)] is that it is a quasi-integrable model that can be analyzed within the framework of the Watanabe-Strogatz theory $[44,45]$. Given a particular initial condition there are $N-3$ constants of motion determining the fate of the system. This degeneracy of the model is not present in (10). Still, the system in Eq. (24) is useful, at least, because of two reasons: (i) We can use it to determine the stability (boundary) of incoherent states analytically, see the next sections; and (ii) it is the starting point for higher-order QPR, see Sec. VI.

\section{Stability of incoherent states}

Equation (24) is the QPR of model (10), irrespective of the number $N$ of oscillators. In this section, we take the thermodynamic limit $(N \rightarrow \infty)$ and analyze the stability boundary of the incoherent states. The analysis requires defining a density $\rho$ such that $\rho(\theta, t) d \theta$ is the fraction of oscillators with phases between $\theta$ and $\theta+d \theta$ at time $t$. Additionally, we impose the normalization condition $\int_{0}^{2 \pi} \rho(\theta, t) d \theta=1$. The Kuramoto order parameter is now $Z=\int_{0}^{2 \pi} \rho(\theta, t) e^{i \theta} d \theta$. The oscillator density $\rho$ obeys the continuity equation because of the conservation of the number of oscillators,

$$
\partial_{t} \rho(\theta, t)+\partial_{\theta}[v(\theta) \rho(\theta, t)]=0 .
$$

This is a nonlinear equation since $v=\dot{\theta}$ depends on $\rho$.

According to Eq. (20), all $\overline{|A|^{n} A}$ are linear combinations of $B$ and $Z$. Therefore, all states with $B=Z=0$ are incoherent states since $\Gamma(0,0)=0$. Obviously, there are infinitely many phase densities compatible with $Z=0$, which rotate uniformly: $\rho_{\text {incoh }}(\theta, t)=\rho_{\text {incoh }}(\theta-\Omega t)$. Notably, it will be shown below that not all incoherent states become unstable simultaneously. of $\rho$,

The analysis proceeds introducing the Fourier expansion

$$
\rho(\theta, t)=\frac{1}{2 \pi} \sum_{m=-\infty}^{\infty} \rho_{m}(t) e^{-i m \theta},
$$

with coefficients $\rho_{0}=1$ and $\rho_{-m}=\rho_{m}^{*}$. Inserting (26) into (25), and noting that $Z=\rho_{1}$, we may rewrite our model (24) in Fourier space,

$$
\begin{aligned}
\dot{\rho}_{m}= & i m \Omega \rho_{m}+\frac{m \kappa}{2}\left[\left(1-i \chi_{0}\right) \Gamma\left(\rho_{1}, B\right) \rho_{m-1}\right. \\
& \left.-\left(1+i \chi_{0}\right) \Gamma^{*}\left(\rho_{1}, B\right) \rho_{m+1}\right], \\
\dot{B}= & i \Omega B+\Lambda\left(B-\rho_{1}\right)+\kappa \Gamma\left(\rho_{1}, B\right) .
\end{aligned}
$$

In light of these equations, it becomes apparent the existence of an infinite set of incoherent solutions characterized by $\rho_{1}=B=0$, and $\rho_{m \geqslant 2}=\hat{\rho}_{m} e^{i m \Omega t}$ with arbitrary $\hat{\rho}_{m \geqslant 2}$. We distinguish between the UIS, corresponding to $\hat{\rho}_{m \neq 0}=0$ and the remaining set of NUISs.

The linear stability of the (N)UIS is determined considering the evolution of infinitesimal perturbations of the form $\rho_{m}=\left(\hat{\rho}_{m}+\delta \rho_{m}\right) e^{i m \Omega t}$ and $B=\delta B e^{i \Omega t}$. The linearization of Eq. (27) turns out to be as follows:

$$
\begin{aligned}
\delta \dot{\rho}_{m}= & \frac{m \kappa}{2}\left[\left(1-i \chi_{0}\right) e^{-i \Omega} \hat{\rho}_{m-1} \vec{\nabla} \Gamma \cdot \vec{\delta}\right. \\
& \left.-\left(1+i \chi_{0}\right) e^{i \Omega} \hat{\rho}_{m+1} \vec{\nabla} \Gamma^{*} \cdot \vec{\delta}\right], \\
\dot{\delta B=} & \Lambda\left(\delta B-\delta \rho_{1}\right)+\kappa \vec{\nabla} \Gamma \cdot \vec{\delta} .
\end{aligned}
$$

The right-hand sides of these equations only include perturbations in the subspace spanned by $\rho_{1}$ and $B$; note the shorthand notation $\vec{\delta}=\left(\delta \rho_{1}, \delta \rho_{1}^{*}, \delta B, \delta B^{*}\right)^{T}$, and the gradients $\vec{\nabla} \Gamma$ defined in this subspace and evaluated at $\rho_{1}=B=0$. We, then, have an infinite set of vanishing eigenvalues corresponding to eigenvectors with $\delta B=\delta \rho_{1}=0$ [46].

Hence, according to Eq. (28), the relevant infinitesimal instabilities develop in the subspace spanned by $\rho_{1}$ and $B$. We are led to analyze the $4 \times 4$ Jacobian matrix ruling the dynamics of $\delta B$ and $\delta \rho_{1}$. In this Jacobian, only the second mode $\hat{\rho}_{2}$ (and $\hat{\rho}_{2}^{*}$ ) is present. Moreover, it can be shown that the stability of all incoherent states can be classified by the value of the amplitude $\left|\hat{\rho}_{2}\right|=Q$. This result was already proved in a particular case [18,47], but QPR shows that it is a general property of the coupling via the mean fields in $\mathcal{C}_{A}$.

Finally, we want to stress that the stability boundaries of the (N)UIS obtained from (28) exactly match those of the original system (10). The reason is that QPR is asymptotically valid in the limit $g(\mathcal{A}) \rightarrow 0$, i.e., where the instabilities take place.

\section{QUASI PHASE REDUCTION FOR STUART-LANDAU OSCILLATORS}

In this paper, we address populations of Stuart-Landau oscillators in detail. Reduction via QPR for other $\lambda-\omega$ oscillators is worked out likewise.

\section{A. Linear coupling: mean field complex Ginzburg-Landau equation}

A simple system to illustrate and test our previous findings is the MF-CGLE presented in Sec. III. Written as in (9) the values of $\Lambda=-2$ and $\chi_{0}=c_{2}$ remain those indicated in Sec. II B, and given that $g(\mathcal{A})=\left(1+i c_{1}\right) \bar{A}$, it is straightforward to obtain $\Gamma(Z, B)=\left(1+i c_{1}\right) B$. Hence, the quasi phase reduced model (24) becomes

$$
\begin{aligned}
\dot{\theta_{j}} & =\kappa \eta|B| \sin \left(\Upsilon-\theta_{j}+\alpha\right), \\
\dot{B} & =-2(B-Z)+\kappa\left(1+i c_{1}\right) B,
\end{aligned}
$$

where $\quad B=|B| e^{i \Upsilon}, \eta \equiv \sqrt{\left(1+c_{2}^{2}\right)\left(1+c_{1}^{2}\right)}, \alpha \equiv \arg [1+$ $\left.c_{1} c_{2}+\left(c_{1}-c_{2}\right) i\right]$. Equation (29) is similar to the Kuramoto-Sakaguchi model [4], but with the phase oscillators coupled through $B$ instead of $Z$. Only in the limit $\kappa \rightarrow 0, B$ approaches $Z$ and the standard first-order phase reduction is recovered [19].

\section{Numerical results: Transient dynamics}

To confirm the correctness of our approach, we compare the transient behavior of the MF-CGLE (9) with its QPR (29). We track the evolution of the mean field $Z=\overline{e^{i \theta}}$ for both 


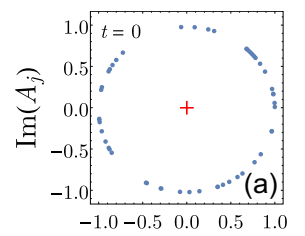

$\operatorname{Re}\left(A_{j}\right)$

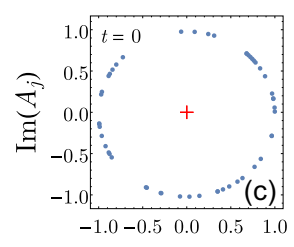

$\operatorname{Re}\left(A_{j}\right)$

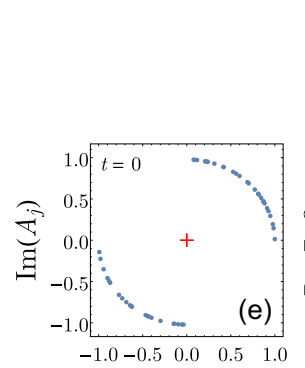

$\operatorname{Re}\left(A_{j}\right)$
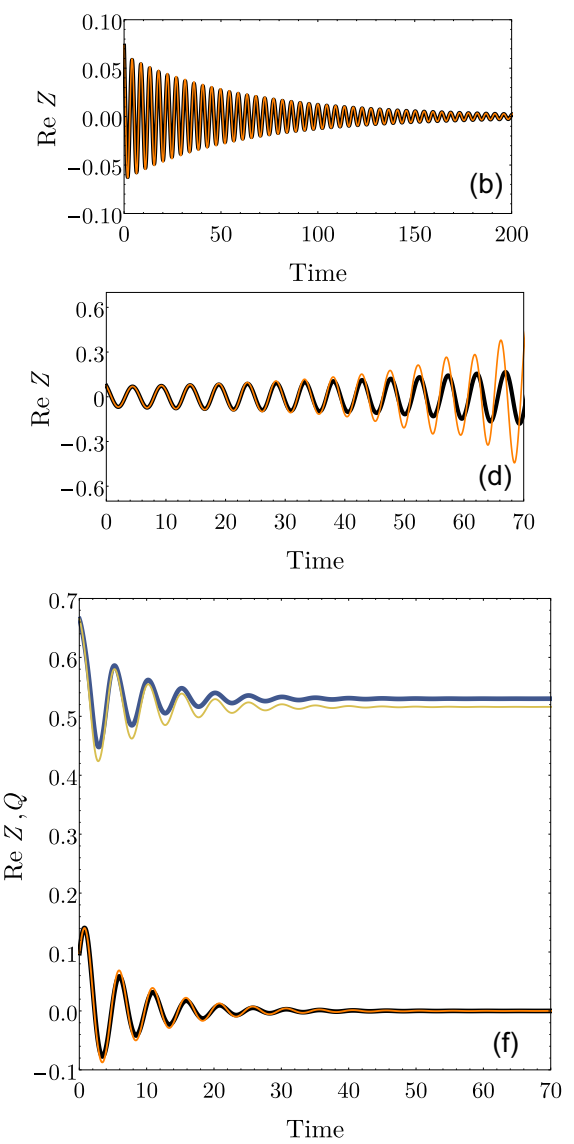

FIG. 2. Time series of the real part of Kuramoto order parameter $Z$, (b), (d), and (f) for the MF-CGLE (9) and its QPR (29) with $N=50$ depicted by black and orange lines, respectively. In (f), the modulus of the second Kuramoto-Daido order parameter $Q$ is also depicted with dark blue and yellow colors for the MF-CGLE and its QPR, respectively. In panels (b) and (d), the oscillators are initially distributed randomly over the unit circle as shown in panels (a) and (c). In (f), the oscillators' phases $\theta_{j}$ are randomly initialized over the interval $\left[0, \frac{\pi}{2}\right] \cup\left[\pi, \frac{3 \pi}{2}\right]$ as shown in panel (e). Accordingly, the system is near the NUIS with $B=Z \simeq 0$ and $Q \simeq 0.66$. The parameters chosen are $c_{2}=3, \kappa=0.5$, and $c_{1}=-1.1$ in (b) where the UIS is stable and $c_{1}=-1$ in (d) and (f) where the UIS is unstable but the NUISs with $Q>\frac{1}{3 \sqrt{2}} \simeq 0.24$ are not.

systems near incoherent states, noting that, for the MF-CGLE, $Z$ is $\overline{e^{i\left(\phi-c_{2} \ln r\right)}}$. In Figs. 2(a)-(d), we initialized $N=50$ oscillators randomly on the unit circle, i.e., near the UIS. The parameters used in Figs. 2(a), 2(b) and 2(c), 2(d) correspond to the stable and unstable UISs, respectively. The stability properties of the UIS (growth rate and oscillation frequency) are perfectly captured by the QPR Eqs. (29). As expected, in Fig. 2(d), after a certain time interval, the mean field $|Z|$ grows too large, and the QPR equations become inaccurate (the MFCGLE approaches a saddle quasiperiodic partial synchrony and eventually decays to a NUIS). In Figs. 2(e) and 2(f), we show that QPR also gives a good description of the NUISs. With the same parameters that in Fig. 2(d), the oscillators were randomly set in the phase interval $\left[0, \frac{\pi}{2}\right] \cup\left[\pi, 3 \frac{\pi}{2}\right]$ of the unit circle. In this way, $B=Z \simeq 0$ but $Q=\left|\overline{e^{i 2 \theta}}\right| \simeq 2 / \pi$. We can see, in Fig. 2(f), that, as time evolves, $Z$ decays to zero, but
$Q$ converges to a nonzero constant value because the UIS is unstable, but the NUISs with large enough $Q$ values are not.

\section{Uniform incoherent state}

A closed formula for the stability boundary of the UIS was already found in Refs. [18,19], so here, we just wish to evidence how QPR permits to obtain it in a simple way. As mentioned above, only the evolution of $\delta \rho_{1}$ and $\delta B$ must be taken into account in Eq. (28). As $\rho_{2}=0$ in the UIS, we get

$$
\frac{d}{d t}\left(\begin{array}{c}
\delta \rho_{1} \\
\delta B
\end{array}\right)=\left(\begin{array}{cc}
0 & \frac{\kappa \eta}{2} e^{i \alpha} \\
2 & -2+\kappa\left(1+i c_{1}\right)
\end{array}\right)\left(\begin{array}{c}
\delta \rho_{1} \\
\delta B
\end{array}\right),
$$

The characteristic equation is as follows:

$$
P_{2}(\lambda)=\lambda^{2}+\left(2-\kappa-i \kappa c_{1}\right) \lambda-\kappa \eta e^{i \alpha}=0 .
$$

The locus of the (oscillatory) instability is determined imposing $\lambda=i \Omega_{c}$. The critical coupling $\kappa_{0}$ satisfies

$$
\kappa_{0}\left(\kappa_{0}-1\right) c_{1}^{2}-4\left(\kappa_{0}-1\right) c_{1} c_{2}+\kappa_{0} c_{2}^{2}+\left(\kappa_{0}-2\right)^{2}=0,
$$

in agreement with Refs. [18,19] (be aware of the different parametrizations in each paper).

\section{Nonuniform incoherent state}

The stability boundary of each NUIS is determined through the evolution of $\delta \rho_{1}$ and $\delta B$. Inserting the specific value of $\hat{\rho}_{2}=Q \leqslant 1$ into Eq. (28) we get

$$
\begin{aligned}
\delta \dot{\rho}_{1} & =\frac{\kappa \eta}{2}\left(e^{i \alpha} \delta B-Q e^{-i \alpha} \delta B^{*}\right), \\
\delta \dot{B} & =-2\left(\delta B-\delta \rho_{1}\right)+\kappa\left(1+i c_{1}\right) \delta B .
\end{aligned}
$$

The associated characteristic polynomial of fourth degree is as follows:

$$
P_{4}(\lambda)=\lambda^{4}+a_{1} \lambda^{3}+a_{2} \lambda^{2}+a_{3} \lambda+a_{4} .
$$

Although the zeros cannot be computed, the Routh-Hurwitz criterion [48] allows to know if there is, at least, one root with nonnegative real part. For the fourth-order polynomial $P_{4}(\lambda)$, all roots have negative real parts if and only if $a_{i}>0$ and $a_{1} a_{2} a_{3}-a_{1}^{2} a_{4}-a_{3}^{2}>0$. This criterion gives five conditions for the stability of a particular " $Q$ NUIS,"

$$
\begin{aligned}
& \kappa_{Q}\left(\kappa_{Q}-1\right) c_{1}^{2}-4\left(\kappa_{Q}-1\right) c_{1} c_{2}+\kappa_{Q} c_{2}^{2}+\left(\kappa_{Q}-2\right)^{2} \\
& +\frac{\kappa_{Q}^{2}\left(1+c_{1}^{2}\right)\left(1+c_{2}^{2}\right)}{\left[\left(2-\kappa_{Q}\right)^{2}+c_{1}^{2}\right]} Q^{2}>0 \\
& 4-2 \kappa_{Q}\left(3 c_{1} c_{2}\right)+\left(1+c_{1}^{2}\right) \kappa_{Q}^{2}>0 \\
& \kappa_{Q}<2
\end{aligned}
$$

plus two other inequalities that are always fulfilled. Equations (32) are precisely the exact $Q$-dependent NUIS stability boundaries of (9) [47].

\section{The effect of arbitrarily weak noise}

A particular (N)UIS may be either unstable or neutrally stable but not asymptotically stable. Thus, in the MF-CGLE, a continuum of neutrally stable incoherent states coexist in regions of parameter space. Hence, the question is the selective effect of arbitrarily weak noise. The color shading in the phase diagram of Fig. 1(a) has been made from Eqs. (30) 


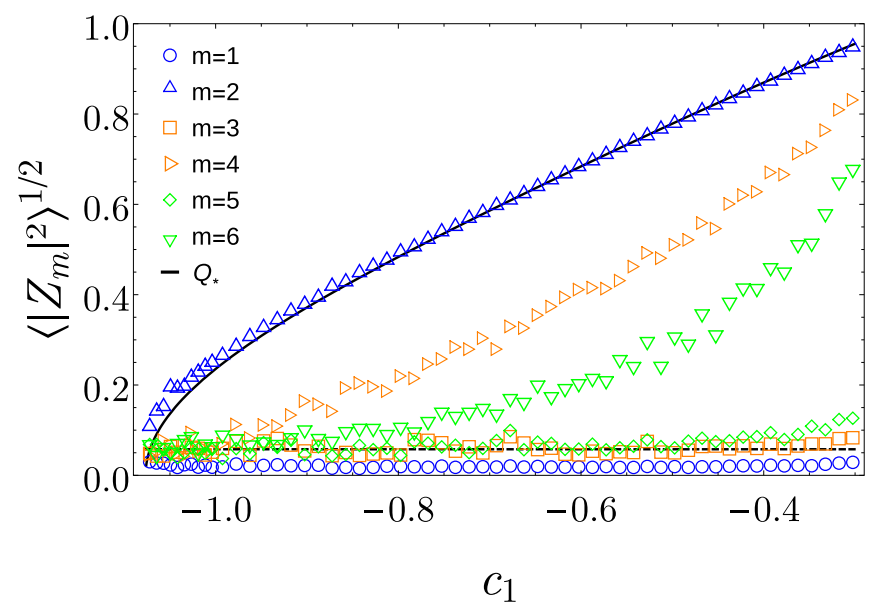

FIG. 3. Root mean square $\left\langle\left|Z_{m}\right|^{2}\right\rangle^{1 / 2}$ of the Kuramoto-Daido order parameters $Z_{m}=\frac{1}{N} \sum_{k=1}^{N} e^{i m \theta_{k}}(m=1, \ldots, 6)$ along the green line in Fig. 1(a) $\left(c_{2}=3, \kappa=0.5\right)$. The black line is $Q_{*}$, the theoretically predicted value of $\left|Z_{2}\right|$, whereas the horizontal dashed line at $1 / \sqrt{N}$ (roughly) indicates the upper expected value of the statistical fluctuations for a vanishing $Z_{m}$ in the thermodynamic limit. The simulations were carried out with $N=300$ oscillators, under independent white Gaussian noises of intensity $D=10^{-6}$ along the real and imaginary parts of the $A_{j}$ 's.

and (32) under the assumption that the system adopts a phase density with $Q=Q_{*}$, where $Q_{*}$ is the smallest $Q$ value among all neutrally stable NUISs. Indeed, a neutral UIS is attracting in the presence of weak noise, see [19]. As may be seen in Fig. 3, in the region where the UIS is unstable, the values of $Q$ observed match almost perfectly with $Q_{*}$, depicted by a black solid line.

Less intuitive is the behavior of the remaining modes, $Z_{m}(m>2)$, that are irrelevant in the stability analysis. According to our Fig. 3 , in the UIS region all $Z_{m}$ 's go to zero, whereas, in the NUIS region, this is only the case for the odd $m$ index. The even modes grow as $Q$ increases. The last NUIS to destabilize is $Q=1$ and corresponds to two equally populated point clusters in antiphase, i.e., a bi-delta phase density $\left(Z_{2}=Z_{4}=Z_{6}=\cdots=e^{i \xi}\right)$.

\section{B. Nonlinear coupling, $g(\mathcal{A}) \propto \overline{|A|^{n} A}$}

Recent papers by Schmidt and co-workers [41,49] have studied chimera states in the MF-CGLE with an extra coupling term proportional to $\overline{|A|^{2} A}$. Here, instead of embarking on the exploration of the high-dimensional parameter space of that system, each coupling of the form $\overline{|A|^{n} A}(n \in \mathbb{Z})$ is analyzed separately. Thus, the systems under consideration are as follows:

$$
\dot{A_{j}}=\left(1+i c_{2}\right)\left(1-\left|A_{j}\right|^{2}\right) A_{j}+\kappa\left(1+i \gamma_{n}\right) \overline{|A|^{n} A},
$$

where the parameter $\gamma_{n}$ is a real constant. In the particular case $n=0$, Eq. (33) becomes the MF-CGLE (9) and $\gamma_{0}=c_{1}$, accordingly.

Deriving the QPR of (33) requires calculating the function $\Gamma(Z, B)$. Using (20) with $\chi_{0}=c_{2}$, the result is straightforward

$$
\Gamma_{n}(Z, B)=\left(1+i \gamma_{n}\right)\left[B+\frac{n}{1+i c_{2}}(B-Z)\right],
$$

where the subscript $n$ is used to indicate the dependence on the specific coupling considered. Finally, inserting $\Gamma_{n}$ into (24) we obtain the QPR of (33),

$$
\begin{aligned}
& \dot{\theta}_{j}=\kappa \eta_{B}|B| \sin \left(\Upsilon-\theta_{j}+\alpha_{B}\right)-\kappa \eta_{R} R \sin \left(\Psi-\theta_{j}+\alpha_{R}\right), \\
& \dot{B}=\Lambda(B-Z)+\kappa \Gamma_{n}(Z, B),
\end{aligned}
$$

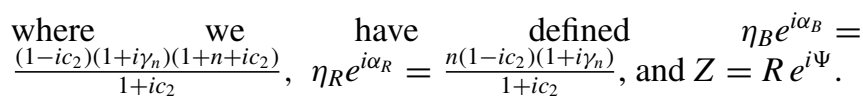

Prior to determining the exact stability boundaries of the (N)UIS from Eq. (35), let us see what the standard first-order phase reduction predicts. For this, we take the limit $\kappa \rightarrow 0^{+}$, observing that $B$ collapses into $Z$, and Eq. (35a) becomes the Kuramoto-Sakaguchi model in Eq. (17). The crossover from perfect synchrony to incoherence is given by the BenjaminFeir-Newell criterion $\left(1+c_{1} c_{2}=0\right)$ in the diffusive case, and, now, generalizes to

$$
1+\gamma_{n} c_{2}=0
$$

by virtue of Eq. (16). Therefore, in a phase diagram of system (33) including the $\kappa$ axis, the stability boundaries of the (N)UIS are expected to emanate from $\gamma_{n}=-c_{2}^{-1}$ at $\kappa=0$.

The exact stability boundaries of the UIS and NUISs in the thermodynamic limit are obtained from (35) as explained in previous sections. The stability boundary of the UIS is as follows:

$$
\begin{aligned}
& \kappa_{0}\left\{4\left(c_{2}-\gamma_{n}\right)^{2}+\gamma_{n}(n+2)\left(\gamma_{n}-c_{2}\right)\right. \\
& \left.\left.\left[\kappa_{0}(n+2)-4\right)\right]\right\}+\left(1+\gamma_{n} c_{2}\right)\left(\kappa_{0}(n+2)-4\right)^{2}=0 .
\end{aligned}
$$

We can see, in the limit $\kappa \rightarrow 0$, we recover (36). The stability boundary of a $Q$-dependent NUIS can be computed as was performed in the linear coupling case; the interested reader can find its expression in the Appendix.

In Fig. 4, the stability boundaries lines of the UIS and NUIS are depicted for five different values of $n=$ $-2,-1, \ldots, 2$. Taking $n=0$ in panel (c) as the reference case, we see that augmenting $n$ shrinks the region of incoherence. On the contrary, for $n=-1$, stable NUISs reach larger $\kappa$ values, whereas the UIS region remains mostly unchanged. The boundaries for other negative $n$ values are similar to those for $n=-2$ in Fig. 4(a). It is interesting that, for all $n$ values, there are regions in parameter space where the UIS is unstable but certain NUISs are not. This means that, at least, for certain initial conditions, the system may spontaneously converge to a NUIS. According to our numerical simulations, and as reasoned above, under weak noise, the nonunstable NUIS with the smallest $Q$ value is observed. In addition, save for $n=-1,0$, there are also regions for small enough $\gamma_{n}$ where the UIS is the last incoherent state to become unstable.

We want to remark that all the stability boundaries calculated are the exact results for (33) and their correctness has been numerically checked using an ensemble of $N=100$ oscillators (not shown). To our knowledge, only the case of the MF-CGLE had been solved so far $[18,47]$. We believe using QPR (35) is the most effective method for computing these boundaries. 

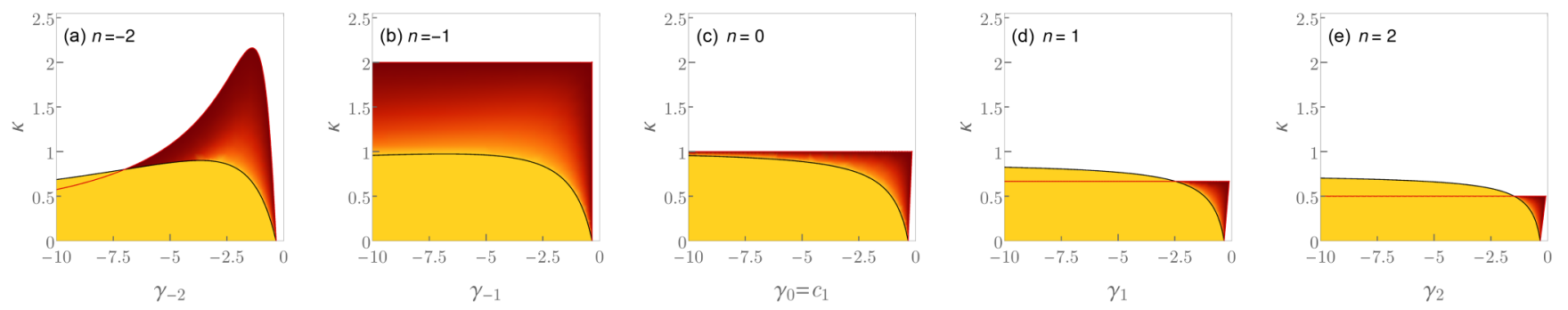

FIG. 4. Partial phase diagrams for populations of Stuart-Landau oscillators with nonisochronicity parameter $c_{2}=3$, coupled through $g(\mathcal{A})=\left(1+i \gamma_{n}\right) \overline{|A|^{n} A}$ with $n=-2,1,0,1,2$, in panels (a)-(e). The stability boundaries of the UIS and the NUIS with $Q=1$ are depicted by black and red lines, respectively. The UIS is stable in the yellow region. In the shaded region, the intensity of the red color indicates $Q_{*}$ (the smallest $Q$ value among the nonunstable NUISs).

\section{Other couplings}

In this subsection, we want to make some comments on the couplings where the $(N+2)$-QPR scheme presented so far can be applied.

It is straightforward to consider a combination of nonlinear couplings, such as

$$
g(\mathcal{A})=\sum_{n=-\infty}^{\infty} \sigma_{n} \overline{|A|^{n} A}+\mu_{n} \overline{|A|^{n} A^{*}},
$$

with complex $\sigma_{n}$ and $\mu_{n}$. In this case, $\Gamma(Z, B)$ is simply a sum over terms, equal to the bracketed part on the right-hand side of Eq. (34), and their complex conjugates.

Other nonlinear coupling considered in Refs. [40,50],

$$
g(\mathcal{A})=\left(\epsilon_{1}+i \epsilon_{2}\right) \bar{A}-\sigma\left(\eta_{1}+i \eta_{2}\right)|\bar{A}|^{2} \bar{A}
$$

can be treated analogously to other couplings. Nonetheless, the stability boundaries of the UIS and NUISs in this case are the same that those for Eq. (9) because the nonlinear term is negligible if $|B|=|\bar{A}| \ll 1$.

Our QPR approach does not exclude systems with nonlinear delayed feedback and/or couplings, such as $h\left(A_{j}\right) g(\mathcal{A})$ (provided $h$ has polar symmetry $h\left(A_{j} e^{i \phi}\right)=e^{i \phi} h\left(A_{j}\right)[51]$ ), similar to those studied in Ref. [52].

Finally, the case of a scalar coupling, such as $g(\mathcal{A}) \propto$ $\operatorname{Re}(\bar{A}) \propto \bar{A}+\bar{A}^{*}$, is particularly simple, as QPR may be further reduced to only one real-valued global variable, i.e., $N+1$ degrees of freedom in total.

\section{EXPLORING THE NEXT ORDER OF QPR}

As it occurs with standard first-order phase reduction, extending the theory to the next order in the QPR scheme is not a trivial task. For QPR, a systematic expansion is even more troublesome as there is not a small coupling parameter but a small field $g(\mathcal{A})$. This should be the goal of future works, but we think it may be instructive to pinpoint the difficulties as well as to examine the workable limit of small coupling.

The first step is to expand Eq. (13) to order $\delta r_{j}$. We obtain, in this way, an augmented version of Eq. (15),

$$
\begin{aligned}
\dot{\theta}_{j}= & \Omega+\kappa \operatorname{Im}\left[\left(1-i \chi_{0}\right) g(\mathcal{A}) e^{-i \theta_{j}}\right] \\
& -\kappa \operatorname{Im}\left\{\left[1+\chi_{0}^{2}+i\left(\chi_{0}+\chi_{1}\right)\right] g(\mathcal{A}) e^{-i \theta_{j}}\right\} \delta r_{j},
\end{aligned}
$$

where $\chi_{1}=\left.\frac{d^{2} \chi(r)}{d r^{2}}\right|_{r=1}$. The deviation from the reference radius $\delta r_{j}$ evolves in time as dictated by Eq. (14), which is coupled to $\theta_{j}$ and to the mean field $\mathcal{A}$. It is not obvious how to proceed next since $\mathcal{A}$ is not static.

\section{A. Small $\kappa$}

Inspecting Eq. (14), we realize that, if $\kappa$ is small, then, $\delta r_{j}(t)$ adjusts quickly to the current mean field,

$$
\delta r_{j}=-\frac{\kappa}{\Lambda} \operatorname{Re}\left[g(\mathcal{A}) e^{-i \theta_{j}}\right]+O\left(\kappa^{2}\right) .
$$

We can insert (39) into (38) to obtain the phase equation up to $O\left(\kappa^{2}\right)$,

$$
\begin{aligned}
\dot{\theta}_{j}= & \Omega+\kappa \operatorname{Im}\left[\left(1-i \chi_{0}\right) g e^{-i \theta_{j}}\right] \\
& +\frac{\kappa^{2}}{\Lambda}\left\{\frac{1+\chi_{0}^{2}}{2} \operatorname{Im}\left(g^{2} e^{-i 2 \theta_{j}}\right)+\left(\chi_{0}+\chi_{1}\right)\left[\operatorname{Re}\left(g e^{-i \theta_{j}}\right)\right]^{2}\right\} .
\end{aligned}
$$

With the new term, proportional to $\kappa^{2}$, the Watanabe-Strogatz theory [44] cannot be applied. This is not a surprise since the original model is not quasi-integrable. To proceed with the analysis, function $g$ has to be written in terms of the mean fields $Z, B$, and maybe others. To the lowest order, we simply adopt the function $\Gamma(Z, B)$ obtained above.

\section{Mean field complex Ginzburg-Landau equation}

Let us see how Eq. (40) applies to the particular case of the MF-CGLE, Eq. (9). As the unit oscillator is the Stuart-Landau oscillator, we insert $\chi_{0}=-\chi_{1}=c_{2}$ into Eq. (40). Moreover, we keep the evolution for $B$ as before. This results in an extended QPR model,

$\dot{\theta}_{j}=\kappa \eta|B| \sin \left(\Upsilon-\theta_{j}+\alpha\right)-\frac{\kappa^{2} \eta^{2}|B|^{2}}{4} \sin \left[2\left(\Upsilon-\theta_{j}\right)+\beta\right]$,

$\dot{B}=-2(B-Z)+\kappa\left(1+i c_{1}\right) B$,

here, $\beta=\arg \left(1-c_{1}^{2}+2 i c_{1}\right)$.

Next, we test (41) by comparing with numerical simulations. We select constants $c_{1}, c_{2}$, and $\kappa$ such that the UIS and full synchrony are both unstable, but the NUISs with $Q$ above a certain value have not destabilized. As observed in Ref, [17], for small and moderate $\kappa$ values, there is a heteroclinic connection between the UIS and a saddle QPS. Recall that, in a QPS state the oscillator density rotates uniformly (as $Z, Z_{2}$, etc., accordingly), but each individual 

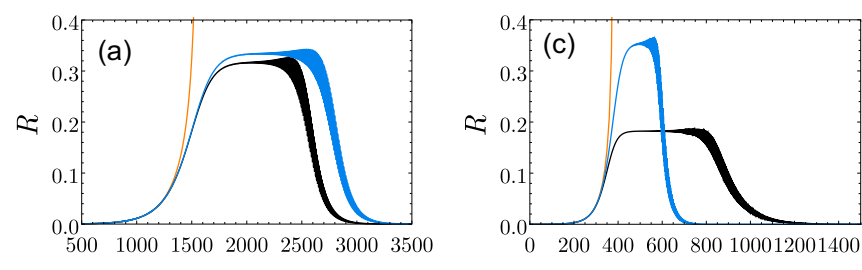

Time
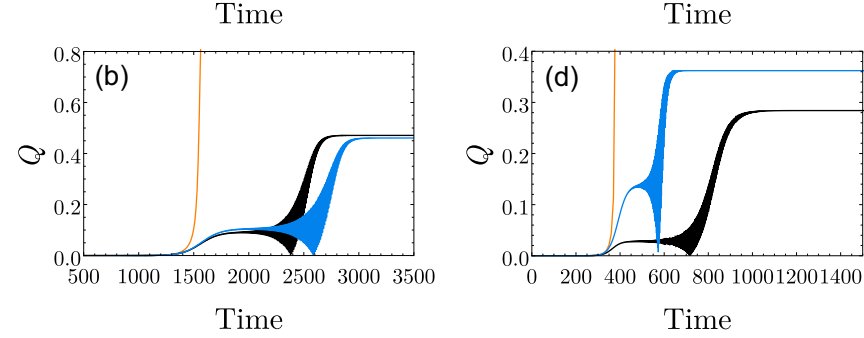

FIG. 5. Time evolution of the MF-CGLE (9) in black, its $(N+$ 2)-QPR (29) in orange, and the extended $(N+2)-\mathrm{QPR}(41)$ in blue. Panels (a)and (c) show the magnitude of the Kuramoto order parameter $R(t)=|Z(t)|$ and (b) and (d) the magnitude of the second Kuramoto-Daido order parameter $Q(t)=\left|Z_{2}(t)\right|$. Two sets of parameters are used: (a) and (b) $c_{1}=-0.5, \kappa=0.2$; (c) and (d) $c_{1}=-1, \kappa=0.5$. Parameter $c_{2}=3$ in all panels.

oscillator exhibits quasiperiodic motion. For the numerical test in Fig. 5, we initialize $N=100$ Stuart-Landau oscillators randomly in the unit circle for the full model (9) as well as the $(N+2)-$ QPR $(29)$ and the extended $(N+2)-\mathrm{QPR}(41)$ with identical initial phases and the $B=Z$ value. Two values of the coupling are selected $\kappa=0.2$ and 0.5 in Figs. 5(a), 5(b) and 5(c), 5(d) respectively. The heteroclinic connection with the saddle QPS is captured by the extended model (41), in contrast to (29), which only reproduces the exponential instability of the UIS. For both, the MF-CGLE (9) and Eq. (41), the final state is a NUIS. Unsurprisingly, the extended QPR (41) is more accurate for $\kappa=0.2$ than for $\kappa=0.5$ since we assumed a small $\kappa$ in its derivation.

For $\kappa$ values larger than those in Fig. 5 there is not a saddle QPS but, instead, a stable QPS branching off from the UIS [28,29]. Remarkably, this also occurs for the extended model (41) at large enough $\kappa$ (not shown).

\section{VII. $(N+2 M)$-QUASI PHASE REDUCTION: HIGHER-ORDER HARMONICS}

In this section, we analyze QPR when we let the oscillators interact through higher-order harmonics,

$$
\dot{A_{j}}=f\left(A_{j}\right)+\kappa g\left(\mathcal{A}^{M}\right),
$$

where $\mathcal{A}^{M}$ are mean fields belonging to the set,

$$
\mathcal{C}_{A}^{M}=\left\{\left\{\overline{|A|^{n} A^{m}}\right\} \cup\left\{\overline{|A|^{n} A^{* m}}\right\}\right\} \underset{\substack{n \in \mathbb{Z} \\ m=1, \ldots, M}}{ } .
$$

These mean fields are the first $M$ harmonics in $\phi$. We show next that, provided the subset of (43) with $m=M$ is not empty, the QPR of Eq. (42) possesses $N+2 M$ degrees of freedom. In other words, the largest harmonic of $\phi$ in the coupling determines the number of degrees of freedom of QPR.
We proceed as in the case $M=1$ seeking to close Eq. (15). For $M>1$, we need to introduce new mean fields $B_{m}=\overline{A^{m}}$ with $m=1, \ldots, M\left(B_{1} \equiv B\right)$. Assuming the $\lambda-\omega$ oscillators are in the neighborhood of the limit cycle at $r=1$, we get

$$
\begin{aligned}
B_{m} & =\overline{A^{m}} \\
& =\overline{r^{m} e^{i m \phi}} \\
& \simeq \overline{(1+m \delta r) e^{i m\left(\theta+i \chi_{0} \delta r\right)}} \\
& \simeq Z_{m}+m\left(1+i \chi_{0}\right) \overline{\delta r e^{i m \theta}},
\end{aligned}
$$

where $Z_{m}=\overline{e^{i m \theta}}$ is the $m$ th Kuramoto-Daido order parameter. We can express $\overline{\delta r e^{i m \theta}}$ in terms of $B_{m}$ and $Z_{m}$,

$$
\overline{\delta r e^{i m \theta}} \simeq \frac{B_{m}-Z_{m}}{m\left(1+i \chi_{0}\right)} .
$$

Applying this equality to the averages $\overline{|A|^{n} A^{m}}$ with an arbitrary $n$ value yields

$$
\begin{aligned}
\overline{|A|^{n} A^{m}} & =\overline{r^{n+m} e^{i m \phi}} \\
& \simeq Z_{m}+\left(n+m+i m \chi_{0}\right) \overline{\delta r e^{i m \theta}} \\
& \simeq B_{m}+\frac{n}{m\left(1+i \chi_{0}\right)}\left(B_{m}-Z_{m}\right) .
\end{aligned}
$$

This relationship permits to approximate $g\left(\mathcal{A}^{M}\right)$ in Eq. (15) in terms of the $M$-dimensional complex vectors $\vec{Z}=$ $\left(Z_{1}, Z_{2}, \ldots, Z_{M}\right)$ and $\vec{B}=\left(B_{1}, B_{2}, \ldots, B_{M}\right)$,

$$
g\left(\mathcal{A}^{M}\right) \simeq \Gamma(\vec{Z}, \vec{B})
$$

The evolution of the phases is, therefore, linked to the set of complex mean fields $\left\{B_{m}\right\}_{m=1, \ldots, M}$, whose evolution equations remain to be determined. Recalling (42), we get

$$
\dot{B}_{m}=m\left[\overline{A^{m-1} f(A)}+\kappa B_{m-1} g\left(\mathcal{A}^{M}\right)\right] .
$$

We see that every $B_{m}$ is influenced by $B_{m-1}$ and by $B_{M}$ (and possibly other $B_{m}$ 's) through $g$, see Eq. (47). The first term on the right-hand side of Eq. (48) is approximated resorting to Eqs. (3), (6), and (46). The result depends only on $B_{m}$ and $Z_{m}$,

$$
\overline{A^{m-1} f(A)}=i \Omega B_{m}+\frac{\Lambda}{m}\left(B_{m}-Z_{m}\right) .
$$

Hence, the $(N+2 M)$-QPR of Eq. (42) is the $(N+2 M)$ dimensional set of ordinary differential equations,

$$
\begin{aligned}
\dot{\theta}_{j} & =\Omega+\kappa \operatorname{Im}\left[\left(1-i \chi_{0}\right) \Gamma(\vec{Z}, \vec{B}) e^{-i \theta_{j}}\right], \\
\dot{B}_{m} & =\Lambda\left(B_{m}-Z_{m}\right)+m\left[i \Omega B_{m}+\kappa \Gamma(\vec{Z}, \vec{B}) B_{m-1}\right]
\end{aligned}
$$

where $j=1, \ldots, N, m=1, \ldots, M$, and $B_{0}=1$.

As a final note, we mention that it is also possible to deal with a coupling function $g(\overline{|A|})$, where $g$ is any function [53].

\section{CONCLUSIONS}

Phase reduction is a powerful technique that has deeply shaped our knowledge on the dynamics of oscillator ensembles. Despite its enormous success, the description enabled by reduced phase models breaks down if the coupling between the oscillators is not weak. Recently, some works have extended standard phase reduction $[17,30,54]$ in a perturbative fashion in the coupling constant. These approaches are, however, condemned to fail for strong coupling. 
In this paper, we have given a new twist to the concept of phase reduction, introducing QPR for all-to-all coupled $\lambda-\omega$ oscillators. This new reduction procedure works independently of the coupling strength, by exploiting the smallness of the collective oscillations near incoherent states. The reduced model has $N+2 M$ degrees of freedom corresponding to $M$ dynamical complex variables mediating the interactions of $N$ phase oscillators, akin to dynamical quorum-sensing models. We have studied in detail the case of $M=1$, corresponding to interactions via the first harmonic of the angle. Explicit stability boundaries for uniform and nonuniform incoherent states have been obtained for ensembles of Stuart-Landau oscillators.

Finally, an extension of QPR beyond the lowest order has been obtained for weak coupling. Nonetheless, a genuine well-controlled expansion to the next order remains to be developed. In parallel with this, some sort of generalization from global to more complex coupling topologies or more general oscillators appears to be possible as well. The case of heterogeneous oscillators-where traditional phase reduction works perfectly (for small couplings) — is amenable to analysis through QPR and will be the aim of future papers. All in all, we deem QPR as a promising path towards a comprehensive theory of collective phenomena in oscillator ensembles.

\section{ACKNOWLEDGMENTS}

We thank J. M. López for proofreading our paper. We acknowledge support by Agencia Estatal de Investigación and Fondo Europeo de Desarrollo Regional under Project No. FIS2016-74957-P (AEI/FEDER, EU). I.L. acknowledges support by Universidad de Cantabria and Government of Cantabria under the Concepción Arenal Programme.

\section{APPENDIX: THE NUIS STABILITY BOUNDARY FOR NONLINEAR COUPLING}

The NUIS stability boundary of (33) is determined using the Routh-Hurwitz criterion and is given by

$$
\begin{aligned}
& 4\left(\gamma_{n}^{2}+1\right)\left(c_{2}^{2}+1\right) \kappa\left(Q^{2}-1\right)[\kappa(n+2)-4]^{2}+[4-\kappa(n+2)]\left[-4 \gamma_{n} c_{2}+\left(\gamma_{n}^{2}+1\right) \kappa\left(n Q^{2}+n+2\right)-4\right]\left(-8 \kappa\left(\gamma_{n} c_{2}+n+3\right)\right. \\
& \left.+\left(\gamma_{n}^{2}+1\right) \kappa^{2}\left\{-\left[n^{2}\left(Q^{2}-1\right)-4 n-4\right]\right\}+16\right)-4 \kappa\left[4 \gamma_{n} c_{2}-\left(\gamma_{n}^{2}+1\right) \kappa\left(n Q^{2}+n+2\right)+4\right]^{2}>0, \\
& \kappa<\frac{4}{2+n}, \\
& \left(\gamma_{n}^{2}+1\right) \kappa^{2}\left\{\left[n^{2}\left(1-Q^{2}\right)+4 n+4\right]\right\}+16-8 \kappa\left(\gamma_{n} c_{2}+n+3\right)>0, \\
& \kappa\left[\left(\gamma_{n}^{2}+1\right) \kappa\left(n Q^{2}+n+2\right)-4 \gamma_{n} c_{2}-4\right]>0 .
\end{aligned}
$$

[1] Y. Kuramoto and H. Nakao, On the concept of dynamical reduction: The case of coupled oscillators, Phil. Trans. R. Soc. A 377, 20190041 (2019).

[2] H. Haken, Synergetics: An Introduction (Springer-Verlag, Berlin, 1983).

[3] J. Guckenheimer and P. Holmes, Nonlinear Oscillations, Dynamical Systems, and Bifurcations of Vector Fields (SpringerVerlag, New York, 1983).

[4] Y. Kuramoto, Chemical Oscillations, Waves, and Turbulence (Springer-Verlag, Berlin, 1984).

[5] H. Nakao, Phase reduction approach to synchronisation of nonlinear oscillators, Contemp. Phys. 57, 188 (2016).

[6] B. Monga, D. Wilson, T. Matchen, and J. Moehlis, Phase reduction and phase-based optimal control for biological systems: A tutorial, Biol. Cybern. 113, 11 (2019).

[7] B. Pietras and A. Daffertshofer, Network dynamics of coupled oscillators and phase reduction techniques, Phys. Rep. 819, 1 (2019).

[8] F. C. Hoppensteadt and E. M. Izhikevich, Weakly Connected Neural Networks (Spinger-Verlag, New York, 1997).

[9] A. T. Winfree, The Geometry of Biological Time (Springer, New York, 1980).

[10] S. H. Strogatz, Sync: The Emerging Science of Spontaneous Order (Hyperion, New York, 2003).
[11] A. Pikovsky and M. Rosenblum, Dynamics of globally coupled oscillators: Progress and perspectives, Chaos 25, 097616 (2015).

[12] A. T. Winfree, Biological rhythms and the behavior of populations of coupled oscillators, J. Theor. Biol. 16, 15 (1967).

[13] Y. Kuramoto, Self-entrainment of a population of coupled non-linear oscillators, in International Symposium on Mathematical Problems in Theoretical Physics, edited by H. Araki, Lecture Notes in Physics Vol. 39 (Springer, Berlin, 1975), pp. $420-422$.

[14] A. S. Pikovsky, M. G. Rosenblum, and J. Kurths, Synchronization, a Universal Concept in Nonlinear Sciences (Cambridge University Press, Cambridge, UK, 2001).

[15] C. van Vreeswijk, Partial synchronization in populations of pulse-coupled oscillators, Phys. Rev. E 54, 5522 (1996).

[16] P. Clusella, A. Politi, and M. Rosenblum, A minimal model of self-consistent partial synchrony, New J. Phys. 18, 093037 (2016).

[17] I. León and D. Pazó, Phase reduction beyond the first order: The case of the mean-field complex Ginzburg-Landau equation, Phys. Rev. E 100, 012211 (2019).

[18] V. Hakim and W. J. Rappel, Dynamics of the globally coupled complex Ginzburg-Landau equation. Phys. Rev. A 46, R7347(R) (1992). 
[19] N. Nakagawa and Y. Kuramoto, Collective chaos in a population of globally coupled oscillators, Prog. Theor. Phys. 89, 313 (1993).

[20] F. Dörfler and F. Bullo, Synchronization in complex networks of phase oscillators: A survey, Automatica 50, 1539 (2014).

[21] K. Wiesenfeld and P. Hadley, Attractor Crowding in Oscillator Arrays, Phys. Rev. Lett. 62, 1335 (1989).

[22] D. G. Aronson, M. Golubitsky, and J. Mallet-Paret, Ponies on a merry-go-round in large arrays of Josephson junctions, Nonlinearity 4, 903 (1991).

[23] L. F. Abbott and C. van Vreeswijk, Asynchronous states in networks of pulse-coupled oscillators, Phys. Rev. E 48, 1483 (1993).

[24] A. Treves, Mean-field analysis of neuronal spike dynamics, Network 4, 259 (1993).

[25] X.-J. Wang and G. Buzsáki, Gamma oscillation by synaptic inhibition in a hippocampal interneuronal network model, J. Neurosci. 16, 6402 (1996).

[26] M. Sinha, F. Dörfler, B. Johnson, and S. Dhople, Phase balancing in globally connected networks of Liénard oscillators, in 2017 IEEE 56th Annual Conference on Decision and Control (CDC), Melbourne, Victoria, Australia, 2017 (IEEE, Piscataway, NJ, 2017), pp. 595-600.

[27] M. Sinha, F. Dörfler, B. Johnson, and S. Dhople, Stabilizing phase-balanced or phase-synchronized trajectories of Van der Pol oscillators in uniform electrical networks, in 2018 56th Annual Allerton Conference on Communication, Control, and Computing (Allerton), Urbana-Champaign, Illinois, 2018 (IEEE, Piscataway, NJ, 2018), pp. 335-340.

[28] N. Nakagawa and Y. Kuramoto, Anomalous Lyapunov spectrum in globally coupled oscillators, Physica D 80, 307 (1995).

[29] P. Clusella and A. Politi, Between phase and amplitude oscillators, Phys. Rev. E 99, 062201 (2019).

[30] D. Wilson and B. Ermentrout, Phase Models Beyond Weak Coupling, Phys. Rev. Lett. 123, 164101 (2019).

[31] O. Castejón, A. Guillamon, and G. Huguet, Phase-amplitude response functions for transient-state stimuli, J. Math. Neurosci. 3, 13 (2013).

[32] D. Wilson and B. Ermentrout, Greater accuracy and broadened applicability of phase reduction using isostable coordinates, J. Math. Biol. 76, 37 (2018).

[33] D. Wilson and B. Ermentrout, Augmented phase reduction of (not so) weakly perturbed coupled oscillators, SIAM Rev. 61, 277 (2019).

[34] N. Kopell and L. N. Howard, Plane wave solutions to reactiondiffusion equations, Stud. Appl. Math. 52, 291 (1973).

[35] M. H. Matheny, M. Grau, L. G. Villanueva, R. B. Karabalin, M. C. Cross, and M. L. Roukes, Phase synchronization of Two Anharmonic Nanomechanical Oscillators, Phys. Rev. Lett. 112, 014101 (2014).

[36] N. Nakagawa and Y. Kuramoto, From collective oscillations to collective chaos in a globally coupled oscillator system, Physica D 75, 74 (1994).
[37] M. Banaji and P. Glendinning, Towards a quasi-periodic mean field theory for globally coupled oscillators, Phys. Lett. A 251, 297 (1999).

[38] K. A. Takeuchi, F. Ginelli, and H. Chaté, Lyapunov Analysis Captures the Collective Dynamics of Large Chaotic Systems, Phys. Rev. Lett. 103, 154103 (2009).

[39] W. L. Ku, M. Girvan, and E. Ott, Dynamical transitions in large systems of mean field-coupled Landau-Stuart oscillators: Extensive chaos and cluster states, Chaos 25, 123122 (2015).

[40] M. Rosenblum and A. Pikovsky, Two types of quasiperiodic partial synchrony in oscillator ensembles, Phys. Rev. E 92, 012919 (2015).

[41] L. Schmidt, K. Schönleber, K. Krischer, and V. García-Morales, Coexistence of synchrony and incoherence in oscillatory media under nonlinear global coupling, Chaos 24, 013102 (2014).

[42] K. Premalatha, V. K. Chandrasekar, M. Senthilvelan, and M. Lakshmanan, Impact of symmetry breaking in networks of globally coupled oscillators, Phys. Rev. E 91, 052915 (2015).

[43] D. J. Schwab, G. G. Plunk, and P. Mehta, Kuramoto model with coupling through an external medium, Chaos 22, 043139 (2012).

[44] S. Watanabe and S. H. Strogatz, Constant of motion for superconducting Josephson arrays, Physica D 74, 197 (1994).

[45] A. Pikovsky and M. Rosenblum, Dynamics of heterogeneous oscillator ensembles in terms of collective variables, Physica D 240, 872 (2011).

[46] The naive expectation is that these neutral modes should decay to zero under arbitrarily weak noise as observed in the UIS of the MF-CGLE [19]. Actually, this is not necessarily the case as a specific example in the next section shows.

[47] M.-L. Chabanol, V. Hakim, and W.-J. Rappel, Collective chaos and noise in the globally coupled complex Ginzburg-Landau equation, Physica D 103, 273 (1997).

[48] F. R. Gantmacher, The Theory of Matrices, Vol. II (Chelsea, New York, 1989).

[49] L. Schmidt and K. Krischer, Clustering as a Prerequisite for Chimera States in Globally Coupled Systems, Phys. Rev. Lett. 114, 034101 (2015).

[50] A. Pikovsky and M. Rosenblum, Self-organized partially synchronous dynamics in populations of nonlinearly coupled oscillators, Physica D 238, 27 (2009).

[51] This condition permits to calculate the equation for $\dot{B}$ using Eq. (20).

[52] O. V. Popovych, C. Hauptmann, and P. A. Tass, Effective Desynchronization by Nonlinear Delayed Feedback, Phys. Rev. Lett. 94, 164102 (2005).

[53] In this case QPR can be accomplished using $\overline{|A|}$ and $\bar{A}$ as the collective variables $(N+3$ degrees of freedom in total).

[54] M. H. Matheny, J. Emenheiser, W. Fon, A. Chapman, A. Salova, M. Rohden, J. Li, M. Hudoba de Badyn, M. Pósfai, L. DuenasOsorio, M. Mesbahi, J. P. Crutchfield, M. C. Cross, R. M. D'Souza, and M. L. Roukes, Exotic states in a simple network of nanoelectromechanical oscillators, Science 363, eaav7932 (2019). 